\title{
Partitioning the vertex set of a bipartite graph into complete bipartite subgraphs
}

\author{
Oleg Duginov"
}

Department of Combinatorial Models and Algorithms, Institute for Mathematics, National Academy of Sciences, Minsk, Belarus

received 30 ${ }^{\text {th }}$ June 2013, revised $31^{\text {st }}$ July 2014, accepted $2^{\text {nd }}$ Sep. 2014.

Given a graph and a positive integer $k$, the biclique vertex-partition problem asks whether the vertex set of the graph can be partitioned into at most $k$ bicliques (connected complete bipartite subgraphs). It is known that this problem is NP-complete for bipartite graphs. In this paper we investigate the computational complexity of this problem in special subclasses of bipartite graphs. We prove that the biclique vertex-partition problem is polynomially solvable for bipartite permutation graphs, bipartite distance-hereditary graphs; and that it remains NP-complete for perfect elimination bipartite graphs and bipartite graphs containing no 4-cycles as induced subgraphs.

Keywords: bicliques, bipartite graph, computational complexity, partitioning problem

\section{Introduction}

In this paper we consider the computational complexity of the problem of partitioning the vertex set of the given graph into at most $k$ bicliques (BICLIQUE VERTEX-PARTITION) in subclasses of bipartite graphs. The problem arises in information security, data mining, e-commerce, information retrieval, and network management [3, 11]. It is known that the BICLIQUE VERTEX-PARTITION problem is NP-complete for bipartite graphs [8, 11] and polynomially solvable for trees [1]. Recently, Martin et al. [15] showed that this problem is computationally hard even for fixed $k=2$.

The aim of this paper is to investigate the following question: which subclasses of bipartite graphs does the BICLIQUe VERTEX-PARTITION problem become polynomially solvable for? In Section 3 we establish a relation between the considered problem restricted to $\left(K_{3}, C_{5}, C_{6}\right)$-free graphs and colouration problems of graphs. In Section 4, using this relation, we show that the Biclique Vertex-PARTition problem is solvable in polynomial time for bipartite permutation graphs and bipartite distance-hereditary graphs. Finally, we establish the NP-completeness of this problem for perfect elimination bipartite graphs and bipartite $C_{4}$-free graphs.

\footnotetext{
*Email: oduginovegmail.com 


\section{Preliminaries}

We use standard graph-theoretic terminology (see, for example, [2, 4, 7]). In this paper we consider only finite undirected simple graphs $G=(V, E)$ with the vertex set $V=V(G)$ and the edge set $E=E(G)$. A graph with the an empty edge set is referred to as an empty graph. We denote the set of all vertices of $G$ adjacent to a vertex $v \in V$ by $N(v)$. For a vertex subset $S \subseteq V$, we denote by $G[S]$ the subgraph of $G$ induced by $S$. If $v \in V$ and $S \subseteq V \backslash\{v\}$, the notation $v \sim S$ means that every vertex in $S$ is adjacent to $v$. A vertex subset $I \subseteq V$ of $G$ is independent if no two vertices in $I$ are adjacent in $G$. A vertex subset $D \subseteq V$ of $G$ is a dominating set if every vertex in $V \backslash D$ is adjacent to at least one vertex in $D$. The minimum size among the dominating sets of $G$ is the domination number of $G$ and is denoted by $\gamma(G)$. A vertex subset $U \subseteq V$ of $G$ is a vertex cover of $G$ if every edge of $G$ is incident with at least one vertex in $U$. The minimum size of a vertex cover of $G$ is the vertex covering number of $G$ and is denoted by $\tau(G)$.

A $u-v$ path of $G$ is a path in $G$ connecting vertices $u, v$ of $G$. The number of edges of a $u-v$ path is its length. The distance $d_{G}(u, v)$ between vertices $u, v$ in $G$ is the length of a shortest $u-v$ path. If $G$ does not contain such a path, then we set $d_{G}(u, v)=\infty$. For a graph $G=(V, E)$ and a positive integer $k$, the $k$ th power of $G$ is the graph $G^{k}$ with the same vertex set $V$ and two vertices $u, v$ are adjacent in $G^{k}$ if and only if $d_{G}(u, v) \leq k$. The complement $\bar{G}$ of a graph $G$ is the graph with vertex set $V(G)$ and $\{u, v\} \in E(\bar{G})$ if and only if $\{u, v\} \notin E(G)$.

For a family $\Gamma$ of graphs, a graph $G$ is $\Gamma$-free if no induced subgraph of $G$ is isomorphic to a graph in $\Gamma$. A graph $G=(V, E)$ is a bipartite graph if there is a partition of the vertex set $V=V_{1} \cup V_{2}$ such that $V_{1}, V_{2}$ are independent sets. For such a bipartite graph $G$ we write $G=\left(\left(V_{1}, V_{2}\right), E\right)$. A graph $G$ is a permutation graph if there are two permutations $P, Q$ of the vertex set $V(G)$ such that there is an edge between vertices $u, v$ if and only if $u$ precedes $v$ in one of $\{P, Q\}$ and $v$ precedes $u$ in the other permutation [18]. A bipartite permutation graph is a permutation graph that is also a bipartite graph. A graph $G$ is distance-hereditary if each connected induced subgraph $F$ of $G$ has the property that $d_{F}(u, v)=d_{G}(u, v)$ for any two vertices $u, v \in V(F)$ [12]. A graph is a bipartite distance-hereditary graph if it is a distance-hereditary graph and a bipartite graph.

A biclique of a graph $G$ is a connected complete bipartite (not necessarily induced) subgraph of $G$. We assume that a subgraph consisting of exactly one vertex is a biclique. A biclique $B=\left(\left(U_{1}, U_{2}\right), E\right)$ is a star centred at a vertex $v$ if $U_{1}=\{v\}$ or $U_{2}=\{v\}$. A set $\mathcal{S}$ of bicliques of $G$ is a biclique vertex-cover of $G$ of size $|\mathcal{S}|$ if each vertex of $G$ is contained in at least one of the bicliques in $\mathcal{S}$. A biclique vertex-cover of $G$ consisting of mutually vertex-disjoint bicliques is a biclique vertex-partition of $G$.

Fleischner et al. [8] observed that one can always make the bicliques of a biclique vertex-cover of a graph vertex-disjoint without increasing the size of the cover. Let $\mathcal{S}=\left\{B_{1}, B_{2}, \ldots, B_{k}\right\}$ be a biclique vertex-cover of a graph $G$ and bicliques $B_{i}, B_{j}(i \neq j)$ have at least one common vertex. Let $S_{i}=$ $V\left(B_{i}\right) \backslash V\left(B_{j}\right), S_{j}=V\left(B_{j}\right) \backslash V\left(B_{i}\right)$ and $S_{i j}=V\left(B_{i}\right) \cap V\left(B_{j}\right)$. If $S_{i}=\emptyset\left(\right.$ or $S_{j}=\emptyset$ ), then the biclique $B_{i}$ (respectively $B_{j}$ ) can be eliminated from the cover $\mathcal{S}$. Thus, we can assume that $S_{i} \neq \emptyset$ and $S_{j} \neq \emptyset$. If $B_{i}-S_{i j}$ is a biclique of $G$, then replace $B_{i}$ in the cover by the biclique $B_{i}-S_{i j}$. Otherwise, there is a vertex $u \in S_{i j}$ such that $u \sim S_{i}$. If $B_{j}$ is not a star centred at $u$, then replace the pair of bicliques $B_{i}, B_{j}$ in the cover $\mathcal{S}$ by vertex-disjoint bicliques $B_{j}-u,\left(\left(\{u\}, S_{i}\right), E_{i}\right)$. In the case when $B_{j}$ is a star centred at $u$, we merge the stars $B_{j}$ and $\left(\left(\{u\}, S_{i}\right), E_{i}\right)$ into one a star, which add to the cover $\mathcal{S}$ instead of the bicliques $B_{i}, B_{j}$. It is not hard to see that after replacing the bicliques $B_{i}$ and $B_{j}$ by at most two vertex-disjoint bicliques, the cover $\mathcal{S}$ remains biclique vertex-cover of $G$ without increasing of the size. This implies the following remark: 
Remark $1([\mathbf{8}])$ For every graph $G$, there is a biclique vertex-cover of $G$ of size at most $k$ if and only if there is a biclique vertex-partition of $G$ of size at most $k$.

For a graph $G$, we denote by $b(G)$ the minimum partition size over all biclique vertex-partitions of $G$. The decision problem associated with the parameter $b(G)$ is defined as follows:

Biclique VERTEX-PARTition

Instance: A graph $G$ and a positive integer $k$.

Question: Does $G$ have a biclique vertex-partition of size at most $k$ ? In other words, is $b(G) \leq k$ ?

The optimisation version of BICLIQUE VERTEX-PARTITION asks for a biclique vertex-partition of the given graph $G$ with minimum size.

\section{Chromatic characterisation of biclique vertex-partitions}

In this section we characterise $b(G)$ for a $\left(K_{3}, C_{5}, C_{6}\right)$-free graph $G$ as the chromatic number of a special vertex colouring of the graph that leads to the construction of a biclique vertex-cover of $G$ such that all vertices of the same colour appear in a single biclique of the cover. It allows us to transform BICLIQUE VERTEX-PARTITION for $\left(K_{3}, C_{5}, C_{6}\right)$-free graphs into an equivalent problem involving vertex colouring of graphs.

Theorem 2 Let $G$ be a $\left(K_{3}, C_{5}\right)$-free graph. If $S \subseteq V(G)$ is a non-empty vertex subset of $G$ such that the induced subgraph $G[S]$ contains at least one edge of $G$ and $d_{G}(u, v) \leq 2$ for any two vertices $u, v$ in $S$, then $G[S]$ is a biclique of $G$.

Proof: Let $G$ be a $\left(K_{3}, C_{5}\right)$-free graph. Assume that $S \subseteq V(G)$ is a non-empty vertex subset of $G$ such that the induced subgraph $G[S]$ contains an edge $e=\{a, b\}$ of $G$ and $d_{G}(u, v) \leq 2$ for any two vertices $u, v$ in $S$.

Let us show that $G[S]$ is a connected graph. Suppose not, and let $w \in S$ be a vertex such that $G[S]$ does not contain any $a-w$ paths and $b-w$ paths. Then the vertices $a, b$ are not adjacent to $w$ in $G$. As $d_{G}(a, w) \leq 2$ and $d_{G}(b, w) \leq 2$, the graph $G$ contains a $a-w$ path and a $b-w$ path that have length 2 . There are only two possibilities: $i$ ) the $a-w$ path and the $b-w$ path have two common vertices and $i i$ ) the $a-w$ path and the $b-w$ path have only one common vertex $w$. Both possibilities are illustrated in Figure 1 . In case $i$ ) the graph $G$ contains $K_{3}$ as an induced subgraph and in case $i i$ ) $G$ contains either $C_{5}$

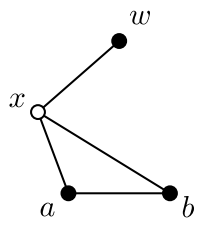

i)

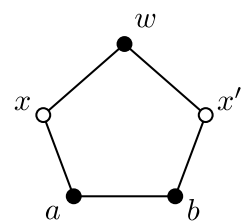

ii)

Fig. 1: In the graph $G$, a $a-w$ path of length 2 and a $b-w$ path of length 2 have either $i$ ) two common vertices, i.e., $w, x$ or $i i$ ) only one common vertex $w$.

(if $\left.\left\{x, x^{\prime}\right\} \notin E(G)\right)$ or $K_{3}$ (if $\left\{x, x^{\prime}\right\} \in E(G)$ ) as an induced subgraph. We obtain a contradiction with 
the assumption that the graph $G$ is a $\left(K_{3}, C_{5}\right)$-free graph. Therefore, $G[S]$ is indeed a connected graph. In fact, we have shown that each vertex $w \in S \backslash\{a, b\}$ is adjacent to at least one vertex in $\{a, b\}$. As a consequence, the graph $G[S]$ does not contain induced subgraphs isomorphic to $\bar{P}_{3}$.

It is well-known that a connected graph is complete bipartite if and only if no induced subgraph is isomorphic to $K_{3}$ or $\bar{P}_{3}$. The connected graph $G[S]$ does not contain induced subgraphs isomorphic to $K_{3}$ (as $G$ doesn't have an induced copy of $K_{3}$ ) or $\bar{P}_{3}$ (as shown above). Therefore, $G[S]$ is indeed a biclique of $G$.

Theorem 3 Let $G$ be a $\left(K_{3}, C_{5}, C_{6}\right)$-free graph. If $S \subseteq V(G)$ is a non-empty vertex subset of $G$ such that $d_{G}(u, v) \leq 2$ for any two vertices $u, v$ in $S$, then the induced subgraph $G[S]$ is either a biclique of $G$ or an empty graph with the condition that there is a vertex $w \in V(G) \backslash S$ such that $w \sim S$.

Proof: Let $G$ be a $\left(K_{3}, C_{5}, C_{6}\right)$-free graph and let $S \subseteq V(G)$ be a non-empty vertex subset of $G$ such that $d_{G}(u, v) \leq 2$ for any two vertices $u, v$ in $S$.

If $|S|=1$ then, by definition, $G[S]$ is a biclique of $G$.

If $|S| \geq 2$ and $G[S]$ contains at least one edge of $G$ then $G[S]$ is a biclique of $G$. It follows from Theorem 2

Consider the case when $|S| \geq 2$ and $G[S]$ does not contain any edges of $G$, i.e., $G[S]$ is an empty graph or, in other words, $S$ is an independent set in $G$. To show that in this case there is a vertex $w \in V(G) \backslash S$ such that $w \sim S$, we use induction on the size of $S$.

Let $S$ consist of only two non-adjacent vertices $u$ and $v$. As $d_{G}(u, v) \leq 2$, the graph $G$ contains a $u-v$ path of length 2. It implies the existence of a vertex $w \in V(G) \backslash S$ adjacent to both $u, v$.

Let $k>2$ be a positive integer number. Assume that for every independent set $S \subseteq V(G)$ of size strictly less than $k$ there exists a vertex $w \in V(G) \backslash S$ such that $w \sim S$ (induction hypothesis). Consider an arbitrary independent set $S \subseteq V(G)$ such that $|S|=k$. We denote by $v_{1}, v_{2}, v_{3}$ three arbitrary vertices in $S$. By the induction hypothesis, there are vertices $w_{1}, w_{2}, w_{3}$ in $V(G) \backslash S$ such that $w_{i} \sim S \backslash\left\{v_{i}\right\}$, $i \in\{1,2,3\}$ (as illustrated in Figure 22. We note that the vertices $w_{1}, w_{2}, w_{3}$ are pairwise non-adjacent

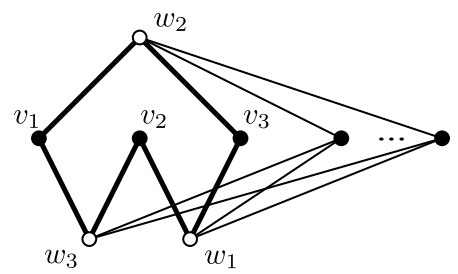

Fig. 2: The set $S$ consists of black vertices. Vertices $w_{1}, w_{2}, w_{3} \in V(G) \backslash S$ such that $w_{i} \sim S \backslash\left\{v_{i}\right\}, i \in\{1,2,3\}$.

(otherwise, the graph $G$ would contain $K_{3}$ as an induced subgraph). Since the graph $G$ does not contain $C_{6}$ as an induced subgraph, there exists $i \in\{1,2,3\}$ such that $w_{i}$ is adjacent to $v_{i}$. Therefore $w_{i} \sim S$ for some $i \in\{1,2,3\}$. It ends the proof of Theorem 3 .

Let $G$ be a graph. A map $c: V(G) \mapsto\{1,2, \ldots, k\}$ is a long-distance colouring of $G$ if $c(u) \neq c(v)$ for any two vertices $u, v$ of $G$ such that $d_{G}(u, v)>2$. The smallest positive integer $k$ such that a graph $G$ has a long-distance colouring $c: V(G) \mapsto\{1,2, \ldots, k\}$ is denoted by $\bar{\chi}(G)$. In other words, $\bar{\chi}(G)$ is the chromatic number of a long-distance colouring of $G$. The following observation is easy to see. 
Observation 4 If $B=\left(\left(U_{1}, U_{2}\right), E\right)$ is a biclique of a graph $G$ then $d_{G}(u, v) \leq 2$ for any two vertices $u, v$ in $U_{1} \cup U_{2}$.

Lemma $5 \bar{\chi}(G) \leq b(G)$ for every graph $G$.

Proof: Let $\left\{B_{1}, B_{2}, \ldots, B_{r}\right\}$ be a biclique vertex-partition of a graph $G$, where every $B_{i}$ is a biclique of $G$. Observation 4 implies that a map $c: V(G) \mapsto\{1,2, \ldots, r\}$ is a long-distance colouring when $c(v)$ is defined as the index $i$ for which $B_{i}$ contains $v \in V(G)$.

Theorem $6 \bar{\chi}(G)=b(G)$ for every $\left(K_{3}, C_{5}, C_{6}\right)$-free graph $G$.

Proof: The inequality $\bar{\chi}(G) \leq b(G)$ was proved in Lemma 5 To show the converse inequality, let $c: V(G) \mapsto\{1,2, \ldots, r\}$ be a long-distance colouring of a $\left(K_{3}, C_{5}, C_{6}\right)$-free graph $G$. Let $V_{i}=\{v \in V(G): c(v)=i\}$. Assume without loss of generality that $V_{i} \neq \emptyset$ for every $i=1,2, \ldots, r$. By Theorem 3, the induced subgraph $G\left[V_{i}\right]$ is either a biclique of $G$ or an empty graph satisfying the condition that there is a vertex $w_{i} \in V(G) \backslash V_{i}$ such that $w_{i} \sim V_{i}$.

If $G\left[V_{i}\right]$ is not a biclique of $G$ then the vertices in $V_{i}$ have a common neighbour $w_{i}$ and $V_{i}$ is included in the vertex set of a $\operatorname{star}\left(\left(\left\{w_{i}\right\}, V_{i}\right), E_{i}\right)$ of $G$. Thus, for each $i=1,2, \ldots, r$ we have a biclique of $G$, say $B_{i}$ that includes all vertices in $V_{i}$. Hence $\left\{B_{1}, B_{2}, \ldots, B_{r}\right\}$ is a biclique vertex-cover of $G$ of size $r$. By Remark 1. if $G$ has a biclique vertex-cover of size at most $r$, then $G$ has a biclique vertex-partition of size at most $r$. Therefore, $b(G) \leq \bar{\chi}(G)$.

As usual, $\chi(G)$ denotes the chromatic number of a graph $G$. For a graph $G$, the complement of the square $\overline{G^{2}}$ has an edge $\{u, v\}$ if and only if $d_{G}(u, v)>2$. Therefore, $\bar{\chi}(G)=\chi\left(\overline{G^{2}}\right)$. The following statement follows directly from Theorem 6

Corollary $7 b(G)=\chi\left(\overline{G^{2}}\right)$ for every $\left(K_{3}, C_{5}, C_{6}\right)$-free graph $G$.

Thus, for every $\left(K_{3}, C_{5}, C_{6}\right)$-free graph $G$ computing $b(G)$ is equivalent to computing $\chi\left(\overline{G^{2}}\right)$. It is known that the chromatic number for perfect graphs is computable in polynomial time [10]. Hence, if $G$ is a $\left(K_{3}, C_{5}, C_{6}\right)$-free graph such that $\overline{G^{2}}$ is perfect, then one can find $b(G)$ in polynomial time, because $\chi\left(\overline{G^{2}}\right)$ can be found in polynomial time. It is well-known that a graph is perfect if and only if its complement is perfect [14]. Summarising these ideas, we obtain the following remark.

Remark 8 Let $\Gamma$ be a graph class. If $\Gamma$ is a subclass of $\left(K_{3}, C_{5}, C_{6}\right)$-free graphs such that $G^{2}$ is perfect for every $G \in \Gamma$, then BICLIQUE VERTEX-PARTITION is polynomially solvable when restricted to $\Gamma$.

\section{Complexity results}

A hierarchy of commonly studied bipartite graphs is given in Figure 3 Previously, the complexity of BICLIQUe VERTEX-PARTITION was known for forests (polynomial) and general bipartite graphs (NPcomplete). In this section, we establish the computational complexity of this problem for bipartite permutation, bipartite distance-hereditary, perfect elimination bipartite, and bipartite $C_{4}$-free graphs. 


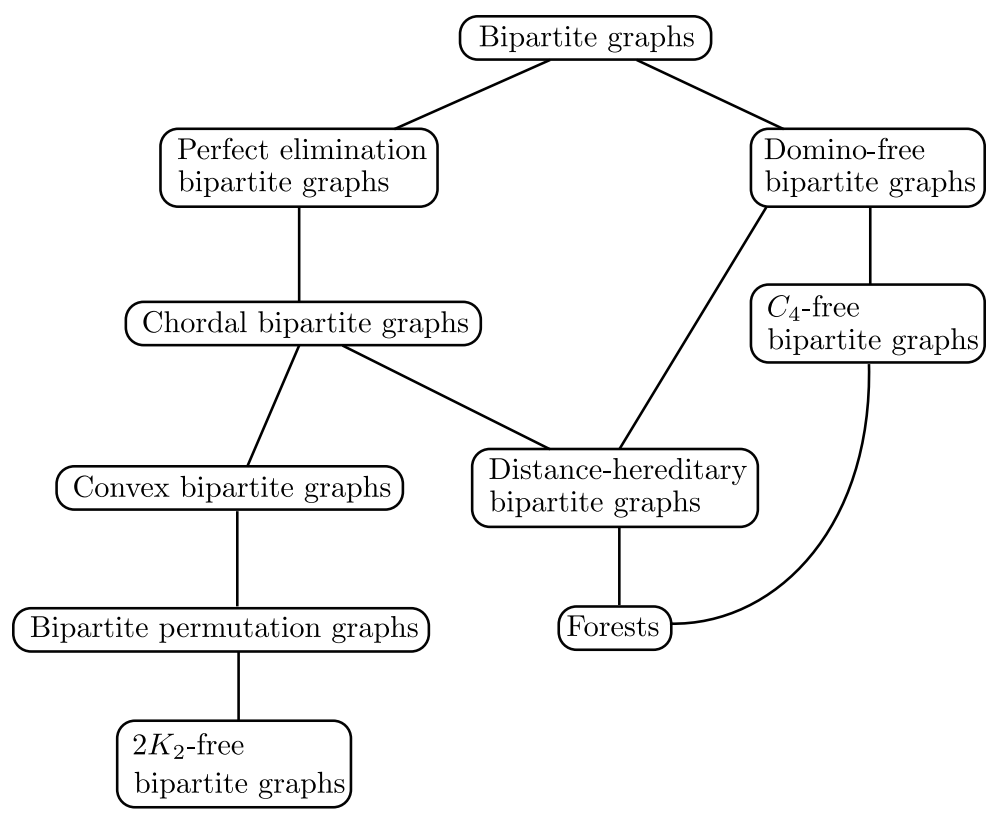

Fig. 3: A hierarchy of bipartite graph classes.

\subsection{Polynomial cases}

We begin with the polynomial cases. BICLIQUE VERTEX-PARTITION is trivially solvable in linear time when restricted to bipartite $P_{4}$-free graphs. Let $G$ be an arbitrary bipartite $P_{4}$-free graph. As every connected $\left(K_{3}, P_{4}\right)$-free graph is complete bipartite, each connected component of $G$ is a biclique of $G$. Therefore, a biclique vertex-partition of $G$ of the minimum size is the set of its connected components, which can be found in linear time by breadth-first search.

Theorem 9 BICLIQUE VERTEX-PARTITION is polynomially solvable for bipartite permutation graphs and bipartite distance-hereditary graphs.

Proof: A bipartite graph is a chordal bipartite graph if each simple cycle of length at least six has a chord, i.e., an edge between two vertices which are not consecutive in the cycle. Thus, every chordal bipartite graph is a $\left(K_{3}, C_{5}, C_{6}\right)$-free graph. As the class of bipartite permutation graphs and the class of bipartite distance-hereditary graphs are subclasses of chordal bipartite graphs, both these graph classes are contained in the class of $\left(K_{3}, C_{5}, C_{6}\right)$-free graphs.

Let us show that $b(G)$ can be found in polynomial time for every bipartite permutation graph $G$. We begin with a definition. A graph is a cocomparability graph if its complement is a comparability graph. Damaschke in [6] showed that the $k$ th power of each cocomparability graph is a cocomparability graph, for all $k \geq 1$. It is known that the class of bipartite permutation graphs is equal to the class of bipartite cocomparability graphs [2, p. 93]. Hence, the square of each bipartite permutation graph is a cocomparability graph. As a consequence of this, $\overline{G^{2}}$ is a comparability graph for every bipartite permutation graph $G$. As mentioned above, the class of bipartite permutation graphs is a subclass of $\left(K_{3}, C_{5}, C_{6}\right)$-free 
graph. By Corollary $7, b(G)=\chi\left(\overline{G^{2}}\right)$ for every bipartite permutation graph $G$. The chromatic number of comparability graphs can be found in linear time [16]. Therefore, $b(G)$ can be found in polynomial time for every bipartite permutation graph $G$ by finding of the chromatic number of the comparability graph $\overline{G^{2}}$.

It is known that even powers of distance-hereditary graphs are chordal [2, p. 164]. Thus, if $G$ is a bipartite distance-hereditary graph then $G^{2}$ is a chordal graph and, consequently, a perfect graph. We conclude that BICLIQUE VERTEX-PARTITION is polynomially solvable for bipartite distance-hereditary graphs; this follows from Remark 8

We note that the well-studied class of convex bipartite graphs is also a subclass of $\left(K_{3}, C_{5}, C_{6}\right)$-free graphs. In view of Corollary $7, b(G)=\chi\left(\overline{G^{2}}\right)$ for every convex bipartite graph $G$. However, there is a convex bipartite graph whose square is not perfect (see Figure 4). So, we cannot show the polynomial

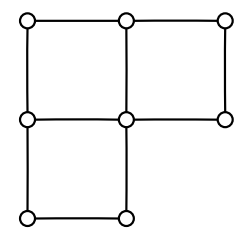

$G$

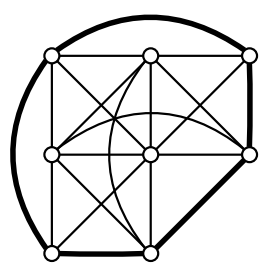

$G^{2}$

Fig. 4: A convex bipartite graph $G$ whose square is not perfect ( $G^{2}$ contains $C_{5}$ as an induced subgraph).

time solvability of BICLIQUE VERTEX-PARTITION for convex bipartite graphs by using Remark 8

\subsection{NP-completeness}

Next, we study the question of whether BICLIQUe VERTEX-PARTITION becomes easier when restricted to bipartite $C_{4}$-free graphs.

Given a dominating set $D$ of a graph $G$, we can partition the vertex set of $G$ using only stars centred at the vertices of $D$. Thus, the domination number $\gamma(G)$ of $G$ is an upper bound on $b(G)$. Let $G$ be a graph containing no $C_{4}$ as a subgraph. As $G$ has no bicliques other than stars, we have $b(G)=\gamma(G)$. Therefore, computing $b(G)$ is equivalent to computing $\gamma(G)$ for all graphs without $C_{4}$ as a subgraph.

Let us observe that every bipartite $C_{4}$-free graph does not contain $C_{4}$ as a subgraph. So, we have $b(G)=\gamma(G)$ for every bipartite $C_{4}$-free graph $G$. Thus, instead of BicLIQUe VerteX-Partition we can consider the decision problem associated with $\gamma(G)$ :

DOMINATING SET

Instance: A graph $G$ and a positive integer $k$.

Question: Does there exist a dominating set of $G$ of size at most $k$ ? In other words, is $\gamma(G) \leq k$ ?

Lemma 10 For any $t \geq 2$, DominATING $\mathrm{SET}$ is NP-complete in the class of 2-connected planar $\left(C_{4}, C_{6}, \ldots, C_{2 t}\right)$-free bipartite graphs of vertex degree at most 6.

Proof: Dominating SeT restricted to 2-connected planar $\left(C_{4}, C_{6}, \ldots, C_{2 t}\right)$-free bipartite graphs of vertex degree at most 6 is trivially in $N P$. For the hardness proof, we use a reduction from the VERTEX 
COVER problem in the class of 2-connected cubic planar graphs, which is known to be NP-complete [17]. Given a graph and a positive integer $k$, VERTEX COVER asks whether there is a vertex cover of the graph of size at most $k$. We closely follow ideas from [5].

Let $t \geq 2$ be an arbitrary integer positive number. Consider an arbitrary odd integer positive number $p \geq t$ such that $p \equiv 1 \bmod 3$. Given a 2-connected cubic planar graph $G=(V, E)$ with $m$ edges, form the graph $G^{\prime}$ by replacing each edge $e=\{u, v\}$ in $G$ by a gadget $G_{e}$ (a simple cycle with $2 p+4$ vertices) illustrated in Figure 5. It is not hard to see that the obtained graph $G^{\prime}$ is a 2-connected planar bipartite

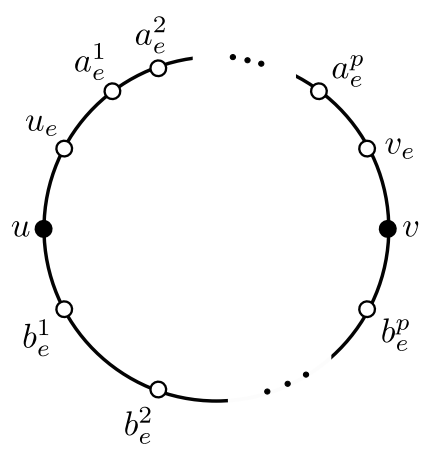

Fig. 5: A gadget $G_{e}$.

graph with maximum degree at most 6 and the graph $G^{\prime}$ does not contain cycles $C_{4}, C_{6}, \ldots, C_{2 t}$ as a subgraphs. We claim that $\gamma\left(G^{\prime}\right)=\tau(G)+\frac{m}{3}(2 p+1)$, where $\tau(G)$ is the vertex covering number of the graph $G$.

Let $S$ be a vertex cover of $G$. We construct a dominating set $D$ of $G^{\prime}$ as follows. Initially set $D=S$. We have that at least one vertex of any two vertices $u, v$ adjacent in $G$ is in $D$. For each edge $e=\{u, v\} \in E$, if $u \in D$ and $v \notin D$ then add to $D$ the following vertices: the vertex $v_{e}$, vertices from minimum dominating set of the $a_{e}^{1}-a_{e}^{p-1}$ path and vertices from minimum dominating set of the $b_{e}^{2}-b_{e}^{p}$ path; otherwise $v \in D$ and in this case add to $D$ the following vertices: the vertex $u_{e}$, vertices from minimum dominating set of the $a_{e}^{2}-a_{e}^{p}$ path and vertices from minimum dominating set of the $b_{e}^{1}-b_{e}^{p-1}$ path. It is easy to see that $D$ is a dominating set of $G^{\prime}$. As $\gamma\left(P_{n}\right)=\left\lceil\frac{n}{3}\right\rceil$ for any $n$ [13],

$$
|D|=|S|+\sum_{e \in E(G)}\left(1+\frac{p-1}{3}+\frac{p-1}{3}\right)=|S|+\frac{m}{3}(2 p+1) .
$$

If $S$ is a vertex cover of $G$ such that $|S|=\tau(G)$ then we obtain that $\gamma\left(G^{\prime}\right) \leq \tau(G)+\frac{m}{3}(2 p+1)$.

Given a dominating set $D$ of $G^{\prime}$, we construct a vertex cover $S$ of $G$ such that $|S| \leq|D|-\frac{m}{3}(2 p+1)$. First construct a dominating set $D^{\prime}$ of $G^{\prime}$ such that $\left|D^{\prime}\right| \leq|D|$ as follows. Initially set $D^{\prime}=D$. Consider an arbitrary edge $e=\{u, v\}$ of $G$ such that $u \notin D^{\prime}, v \notin D^{\prime}$ and let $D_{e}^{a}=D^{\prime} \cap\left\{u_{e}, v_{e}, a_{e}^{1}, a_{e}^{2}, \ldots, a_{e}^{p}\right\}$, $D_{e}^{b}=D^{\prime} \cap\left\{b_{e}^{1}, b_{e}^{2}, \ldots, b_{e}^{p}\right\}$. As $u \notin D^{\prime}, v \notin D^{\prime}$ and $p \equiv 1 \bmod 3$,

$$
\left|D_{e}^{a}\right| \geq \gamma\left(P_{p+2}\right)=\frac{p+2}{3}, \quad\left|D_{e}^{b}\right| \geq \gamma\left(P_{p}\right)=\left\lceil\frac{p}{3}\right\rceil=\frac{p+2}{3} .
$$

In this case, replace $D_{e}^{a} \cup D_{e}^{b}$ in $D^{\prime}$ by a set $R_{e}$ consisting of the vertices $u, v_{e}$, vertices from minimum dominating set of the $a_{e}^{1}-a_{e}^{p-1}$ path and vertices from minimum dominating set of the $b_{e}^{2}-b_{e}^{p}$ path, i.e., 
we set $D^{\prime}=\left(D^{\prime} \backslash\left(D_{e}^{a} \cup D_{e}^{b}\right)\right) \cup R_{e}$. We note that after applying this transformation to $D^{\prime}$, it remains a dominating set of $G^{\prime}$ without increasing its size. After transforming of $D^{\prime}$ for each $e=\{u, v\} \in E$ such that $u \notin D^{\prime}$ and $v \notin D^{\prime}$ one after another, we obtain a dominating set $D^{\prime}$ of $G^{\prime}$ such that $\left|D^{\prime}\right| \leq|D|$. It is not hard to see that for each $e=\{u, v\} \in E$ at least one of the vertices $u, v$ is contained in $D^{\prime}$ and

$$
\left|D^{\prime} \cap\left\{u_{e}, v_{e}, a_{e}^{1}, a_{e}^{2}, \ldots, a_{e}^{p}, b_{e}^{1}, b_{e}^{2}, \ldots, b_{e}^{p}\right\}\right| \geq \gamma\left(P_{p}\right)+\gamma\left(P_{p-2}\right)=\frac{2 p+1}{3} .
$$

Therefore, the set $S=D^{\prime} \cap V$ is a vertex cover of $G$ and $\left|D^{\prime}\right| \geq|S|+m \frac{2 p+1}{3}$. Hence

$$
|D| \geq\left|D^{\prime}\right| \geq|S|+m \frac{2 p+1}{3} .
$$

If $D$ is a dominating set of $G^{\prime}$ such that $|D|=\gamma\left(G^{\prime}\right)$ then we obtain $\gamma\left(G^{\prime}\right) \geq \tau(G)+m \frac{2 p+1}{3}$.

Thus, $\gamma\left(G^{\prime}\right)=\tau(G)+\frac{m}{3}(2 p+1)$ and $G$ has a vertex cover of size at most $k$ if and only if $G^{\prime}$ has a dominating set of size at most $k+\frac{m}{3}(2 p+1)$, where $m$ is the number of edges of $G$.

As for every bipartite $C_{4}$-free graph $G$ computing $b(G)$ is equivalent to computing $\gamma(G)$, the following statement is a direct consequence of Lemma 10 .

Corollary 11 For any $t \geq 2$, BICLIQUE VERTEX-PARTITION is NP-complete for 2-connected planar $\left(C_{4}, C_{6}, \ldots, C_{2 t}\right)$-free bipartite graphs of vertex degree at most 6 . In particular, BICLIQUE VERTEXPARTITION remains $N P$-complete for bipartite $C_{4}$-free graphs.

Now we study the computational complexity of BICLIQUE VERTEX-PARTITION for perfect elimination bipartite graphs. Let $G=((X, Y), E)$ be a bipartite graph. An edge $e=\{x, y\} \in E$ is a bisimplicial edge if $N(x) \cup N(y)$ induces a biclique of $G$. Let $\left(e_{1}, e_{2}, \ldots, e_{k}\right)$ be a sequence of pairwise non-adjacent edges of $G$ (not necessarily all edges of $E$ ). We denote by $S_{i}$ the set of endpoints of the edges $e_{1}, e_{2}, \ldots, e_{i}$ and $S_{0}=\emptyset$. The sequence $\left(e_{1}, e_{2}, \ldots, e_{k}\right)$ is a perfect edge elimination ordering for $G$ if $G\left[(X \cup Y) \backslash S_{k}\right]$ is an empty graph and each edge $e_{i}$ is a bisimplicial edge in $G\left[(X \cup Y) \backslash S_{i-1}\right]$. The graph $G$ is a perfect elimination bipartite graph (see, for example, [2, p. 87], [9]) if $G$ has a perfect edge elimination ordering.

Theorem 12 BICLIQUE VERTEX-PARTITION is NP-complete for perfect elimination bipartite graphs.

Proof: We make a simple reduction from BICLIQUe VERTEX-PARTITION, which is known to be NPcomplete for bipartite graphs. Suppose we are given a bipartite graph $G=((X, Y), E)$ with $X=\left\{x_{1}, x_{2}, \ldots, x_{p}\right\}, Y=\left\{y_{1}, y_{2}, \ldots, y_{q}\right\}$ and $p \geq 1$. We transform $G$ into a bipartite graph $G^{\prime}=\left(\left(X^{\prime}, Y^{\prime}\right), E^{\prime}\right)$, where $X^{\prime}=X \cup\left\{b_{1}, b_{2}, \ldots, b_{p}\right\}, Y^{\prime}=Y \cup\left\{a_{1}, a_{2}, \ldots, a_{p}\right\} \cup\left\{c_{1}, c_{2}, \ldots, c_{p}\right\}$ and

$$
E^{\prime}=E \cup \bigcup_{i=1}^{p}\left\{\left\{x_{i}, a_{i}\right\},\left\{a_{i}, b_{i}\right\},\left\{b_{i}, c_{i}\right\}\right\} .
$$

Thus, $G^{\prime}$ is obtained from $G$ by appending a path $\left\{x_{i}, a_{i}, b_{i}, c_{i}\right\}$ to $x_{i}$ for every $i=1,2, \ldots, p$ (see Figure 6 for an example). The graph $G^{\prime}$ is a perfect elimination bipartite graph. The sequence

$$
\sigma=\left(\left\{b_{1}, c_{1}\right\},\left\{b_{2}, c_{2}\right\}, \ldots,\left\{b_{p}, c_{p}\right\},\left\{x_{1}, a_{1}\right\},\left\{x_{2}, a_{2}\right\}, \ldots,\left\{x_{p}, a_{p}\right\}\right)
$$

is a perfect edge elimination ordering for $G^{\prime}$. We claim that $b\left(G^{\prime}\right)=b(G)+p$. 


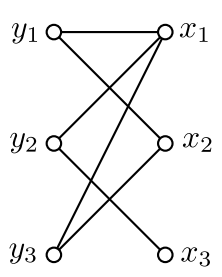

$G$

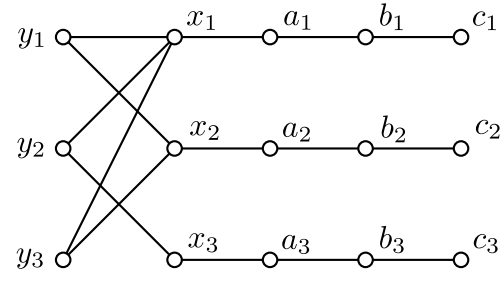

$G^{\prime}$

Fig. 6: A bipartite graph $G$ and the graph $G^{\prime}$ obtained from $G$.

For each $i=1,2, \ldots, p$, we denote by $B_{i}$ the star of $G^{\prime}$ centred at the vertex $b_{i}$ that contains the vertices $b_{i}$ and $c_{i}$, i.e., $B_{i}=\left(\left(\left\{b_{i}\right\},\left\{a_{i}, c_{i}\right\}\right),\left\{\left\{b_{i}, a_{i}\right\},\left\{b_{i}, c_{i}\right\}\right\}\right)$. Let $\mathcal{S}$ be a biclique vertex-partition of $G$. Form $\mathcal{S}^{\prime}$ by adding the stars $B_{1}, B_{2}, \ldots, B_{p}$ to $S$. It is easy to see that $S^{\prime}$ is a biclique vertex-partition of $G^{\prime}$ of size $|\mathcal{S}|+p$. Taking $\mathcal{S}$ with the size $b(G)$, we obtain $b\left(G^{\prime}\right) \leq b(G)+p$. To show the converse inequality, we use the following simple observation.

Observation 13 The graph $G^{\prime}$ has a biclique vertex-partition $\mathcal{S}^{\prime}$ of size $b\left(G^{\prime}\right)$ that contain the stars $B_{1}, B_{2}, \ldots, B_{p}$.

Let $\mathcal{S}^{\prime}$ be a biclique vertex-partition of $G^{\prime}$ from Observation 13 . Construct $\mathcal{S}$ from $\mathcal{S}^{\prime}$ by eliminating the stars $B_{1}, B_{2}, \ldots, B_{p}$. It is easy to see that $\mathcal{S}$ is a biclique vertex-partition of $G$ of size $b\left(G^{\prime}\right)-p$. Hence $b(G) \leq b\left(G^{\prime}\right)-p$.

Thus, we have that $b\left(G^{\prime}\right)=b(G)+p$ and, as a consequence, the bipartite graph $G$ has a biclique vertex-partition of size at most $k$ if and only if the perfect elimination bipartite graph $G^{\prime}$ has a biclique vertex-partition of size at most $k+p$.

\section{Conclusion and further work}

In this paper, we have shown that the BICLIQUE VERTEX-PARTITION problem is NP-complete for perfect elimination bipartite graphs and have provided two subclasses of perfect elimination bipartite graphs for which this problem is solvable in polynomial time. To refine the borderline between polynomial time solvability and NP-completeness, it would be interesting to establish the computational complexity of the given graph problem for convex bipartite graphs and for chordal bipartite graphs.

We have also shown that the BICLIQUE VERTEX-PARTITION problem remains NP-complete even for bipartite $C_{4}$-free graphs. It would be interesting to find subclasses of bipartite $C_{4}$-free graphs for which the considered problem is polynomially solvable.

\section{Acknowledgements}

I would like to thank Victor V. Lepin for fruitful discussions and the anonymous referees for comments and suggestions. This research was supported by Belarusian Republican Foundation for Fundamental Research (project F12RA-006). 


\section{References}

[1] D. Bein, L. Morales, W. Bein, Z. Meng, C.O. Shields Jr., and I.H. Sudborough. Clustering and the biclique partition problem. In Proceedings of the 41st Annual Hawaii International Conference on System Sciences (HICSS 2008), page 475, 2008.

[2] A. Brandstädt, V.B. Le., and J. Spinrad. Graph classes: a survey. SIAM, Philadelphia, 1999.

[3] D. Chakrabarti, S. Papadimitriou, D.S. Modha, and C. Faloutsos. Fully automatic cross-associations. In Proceedings of the tenth ACM SIGKDD international conference on Knowledge discovery and data mining, pages 79-88, 2004.

[4] G. Chartrand and L. Lesniak. Graphs and Digraphs. Chapman \& Hall/CRC, Boca Raton, 4th edition, 2005.

[5] M. Chlebik and J. Chlebiková. Approximation hardness of dominating set problems in bounded degree graphs. Information and Computation, 206:1264-1275, 2008.

[6] P. Damaschke. Distances in cocomparability graphs and their powers. Discr. Appl. Math., 35:67-72, 1992.

[7] R. Diestel. Graph Theory. Springer, New-York, 2nd edition, 2000.

[8] H. Fleischner, E. Mujuni, D. Paulusma, and S. Szeider. Covering graphs with few complete bipartite subgraphs. Theor. Comput. Sci., 410:2045-2053, 2009.

[9] M.C. Golumbic and C.F. Goss. Perfect elimination and chordal bipartite graphs. Journal of Graph Theory, 2:155-163, 1978.

[10] M. Grötschel, L. Lovász, and A. Schrijver. Polynomial algorithms for perfect graphs. Ann. Discrete Math., 21:325-356, 1984.

[11] M.H. Heydari, L. Morales, C.O. Shields Jr., and I.H. Sudborough. Computing cross associations for attack graphs and other applications. In Proceedings of 40th Annual Hawaii International Conference on System Sciences (HICSS 07), page 270, 2007.

[12] E. Howorka. A characterization of distance-hereditary graphs. The Quarterly Journal of Mathematics, 28:417-420, 1977.

[13] M.S. Jacobson and L.F. Kinch. On the domination number of products of graphs: I. Ars Combinatoria, 18:33-44, 1983.

[14] L. Lovász. Normal hypergraphs and the perfect graph conjecture. Discr. Math., 2:253-267, 1972.

[15] B. Martin and D. Paulusma. The computational complexity of disconnected cut and $2 \mathrm{~K}_{2}-$ partition. In Principles and Practice of Constraint Programming - CP2011, pages 561-575, 2011.

[16] R. McConnell and J. Spinrad. Linear-time transitive orientation. In Proceedings of the eighth annual ACM-SIAM symposium on Discrete algorithms, pages 19-25, 1997. 
[17] B. Mohar. Face covers and the genus problem for apex graphs. Journal of Combinatorial Theory, Series B, 82:102-117, 2001.

[18] J. Spinrad, A. Brandstädt, and L. Stewart. Bipartite permutation graphs. Discrete Applied Mathematics, 18:279-292, 1987. 